\title{
Unraveling the opioid actions of S-ketamine and R-ketamine: comment on Bonaventura et al.
}

\author{
Boris D. Heifets ${ }^{1,2} \cdot$ Brandon S. Bentzley $^{2} \cdot$ Nolan Williams $^{2} \cdot$ Alan F. Schatzberg $\mathbb{B}^{2}$
}

Received: 15 April 2021 / Revised: 29 April 2021 / Accepted: 6 May 2021 / Published online: 18 May 2021

(c) The Author(s), under exclusive licence to Springer Nature Limited 2021

If only ketamine were as well-behaved a drug as citalopram. Although our understanding of how selective serotonin reuptake inhibitors (SSRIs) relieve symptoms of depression continues to evolve, the similar efficacy of myriad other SSRIs indicates that the process begins with an increase in synaptic serotonin. With this basic understanding of drug mechanism, citalopram's success was followed by commercialization of the active enantiomer responsible for its SSRI properties. Although all SSRIs have roughly the same efficacy in treating depression, the purified enantiomer of citalopram, escitalopram, is more selective for its target allowing for therapeutic doses that largely avoid adverse effects on cardiac conduction. In contrast, the story for ketamine is considerably more complicated and potentially more pressing to understand, given ketamine's rapidly growing use in clinics and its well-known abuse potential.

Research over the past several years has dramatically broadened the scope of possible explanations for ketamine's antidepressant mechanism(s), including a renewed interest in its enantioselective actions, its metabolites, with all of their diastereomeric complexity, and a host of overlapping and potentially interacting molecular targets. Despite considerable early enthusiasm for N-Methyl D-Aspartate receptor (NMDAR) antagonism as the primary antidepressant mechanism for ketamine, this explanation seems incomplete, considering that clinical trials of other NMDAR antagonists have failed to reproduce the rapid and sizeable antidepressant effect of ketamine [1-4].

In this issue of Molecular Psychiatry, Bonaventura et al bring some order to the chaos surrounding ketamine

Alan F. Schatzberg

afschatz@stanford.edu

1 Department of Anesthesiology, Perioperative and Pain Medicine, Stanford University School of Medicine, Stanford, CA, USA

2 Department of Psychiatry and Behavioral Sciences, Stanford University School of Medicine, Stanford, CA, USA through systematic and rigorous parallel assessments of (S)and (R)-ketamine at multiple levels, spanning molecular pharmacology, in vivo nuclear imaging, and murine behavioral assays. We are not surprised to see that out of nearly 100 screened receptors and enzymes, the authors found that ketamine interacts significantly with mu opioid receptors (MORs) and this interaction is comparable to ketamine's interaction with NMDARs. This finding echoes our result in humans where an opioid antagonist, naltrexone, blocked the antidepressant effect of ketamine [5, 6]. The extensive screening and biased molecular signaling assays reported by the authors bring much-needed modern methods to bear on the question of ketamine's interaction with the opioid signaling pathways; yet, it should be noted that many of these basic findings have been known since at least 1978 [7-9]. The authors' data build upon these earlier works and strongly point to a direct interaction between ketamine and the MOR, although indirect interactions such as ketaminestimulated endogenous opioid release may also occur [10].

Most notably, the authors dispel the myth that ketamine has meaningful selectivity for NMDARs over other targets, such as opioid receptors, at behaviorally relevant doses. In an unbiased screen against a substantial fraction of the human proteome, no high affinity targets were identified. Similarly, nuclear imaging did not reveal any significant high affinity binding of ketamine to brain tissue. On the other hand, nuclear PET imaging revealed that behaviorally relevant doses of ketamine, in particular (S)-ketamine, had enantioselective, opioid-receptor dependent effects on regional brain metabolism, as indexed by ${ }^{18}$ FDG.

How these pharmacological and physiological effects translate into behavior that can be modeled in a rodent, and ultimately into a coherent understanding of ketamine's antidepressant mechanism, is less clear. This study explored the relative reinforcing properties of ketamine enantiomers, and the authors demonstrated that (S)-ketamine preferentially activates the MOR compared to (R)-ketamine. As would be expected, rats preferentially self-administered the (S)-enantiomer, developed a preference for a context associated with 
the (S)- enantiomer, and developed locomotor sensitization to the (S)-enantiomer; whereas, the same behaviors were not observed for the (R)- enantiomer. However, it remains unclear whether these differences in behavioral effects are a result of MOR signaling. The authors report that locomotor stimulating effects of (S)-ketamine are blocked by naltrexone, but we are left to wonder whether the same is true for the drug reward behaviors they report on, conditioned place preference and drug self-administration. To make matters even more confusing, the behavioral data do not align with the ketamine enantiomers' effects on dopamine. The authors evaluated another physiological proxy for drug reward, dopamine release, by measuring regional uptake of ${ }^{18} \mathrm{~F}$-fallypride, a dopamine receptor ligand that can be displaced by stimulating release of dopamine. Based on the behavioral data, one might expect that the (S) enantiomer would be associated with greater dopamine release in the most wellstudied reward center in the brain, the nucleus accumbens (NAc). In fact, the opposite was observed: (R)-ketamine preferentially reduced ${ }^{18} \mathrm{~F}$ fallypride uptake in the NAc, whereas (S)-ketamine appeared to stimulate more dopamine release in the prefrontal cortex and other areas.

On the whole, the behavioral data speak for themselves. (S)-ketamine appears to be responsible for ketamine's drug reward properties. The obvious question to move drug discovery forward is how do these findings map onto ketamine's antidepressant mechanisms of action, and can the therapeutic and abuse-linked properties be separated? It is as yet unclear whether intranasal esketamine (Spravato ${ }^{\circledast}$ ) has the same antidepressant efficacy as racemic ketamine, noting the preclinical and preliminary clinical data suggesting superior efficacy of the (R) enantiomer, as cited in this study. (R)-ketamine may in fact have less abuse liability and toxicity, but it may in the end be substantially less effective or less rapidly effective than esketamine. In fact, there is no reason to believe that ketamine's antidepressant mechanism can be reduced to a single molecular interaction - as we and others have suggested, some molecular interactions, e.g., with opioid receptors, may drive the rapid antidepressant effect, while others may be responsible for the days-to-weeks long durability [5]. More recently Klein and colleagues [11] have reported that the opioid effects of racemic ketamine are necessary but not sufficient for its antidepressant effects. Formal testing with detailed time courses in humans for each component of ketamine is the way forward. In addition, these data suggest that other agents purported to produce antidepressant-like effects in rodents via NMDA antagonism should be studied for their ability to bind to MORs or to mobilize endogenous opioid release. Inability to affect opioid activity may turn out to augur limited antidepressant effects in humans.

Answering these questions is important and may have public health consequences. Ketamine has a well-documented rapid antidepressant effect when given once, and many early trials focused on its efficacy as a single dose treatment. That is not how it is being used in a growing number of community practices [12], where patients commonly receive a series of 4-6 (or more) induction doses over a two-week period, followed by maintenance treatments that do not have a welldefined frequency or endpoint. More clinical data will likely tell us how much and how often ketamine should be administered to achieve response and remission [13, 14]. The need for ongoing ketamine maintenance therapy represents a major limitation to its widespread use given the well-known abuse potential of ketamine, as well as the potential toxicity associated with long term use. Although recognition of these risks is emerging in the United States [15], ketamine abuse and illicit trafficking is a recognized public health issue worldwide $[16,17]$ to an extent that in 2015 the Government of China proposed reclassifying ketamine into the most restrictive category (Schedule 1) under international regulations. Moreover, long term use of ketamine has been associated with significant tolerance, gastrointestinal and urologic toxicity as well as neurocognitive impairment [18-21], side effects with an as-yet unknown impact on patients receiving ongoing ketamine maintenance therapy. For all these reasons, a clear mechanistic understanding of how ketamine, its enantiomers, and its metabolites work in the brain is the best way forward to developing better, safer therapeutics for depression.

Acknowledgements BDH received an NIH K08 Career Development Award through the NIMH (MH110610). AFS work was supported by funding from the American Foundation for Suicide Prevention and the Pritzker Foundation.

\section{Compliance with ethical standards}

Conflict of interest BDH and BSB have no relevant disclosures. NW has served on a scientific advisory board for Halo Neuroscience, Neurawell, Nooma, and Otsuka. AFS discloses he has in the past 3 years received research funding and consulting fees from Janssen and consulting fees from Epiodyne.

Publisher's note Springer Nature remains neutral with regard to jurisdictional claims in published maps and institutional affiliations.

\section{References}

1. Zarate CA, Singh JB, Quiroz JA, De Jesus G, Denicoff KK, Luckenbaugh DA, et al. A double-blind, placebo-controlled study of memantine in the treatment of major depression. Am J Psychiatry. 2016;163:153-5.

2. Heresco-Levy U, Javitt DC, Gelfin Y, Gorelik E, Bar M, Blanaru $\mathrm{M}$, et al. Controlled trial of D-cycloserine adjuvant therapy for treatment-resistant major depressive disorder. J Affect Disord. 2016;93:239-43.

3. Paterson B, Fraser H, Wang C, Marcus R. A randomized, doubleblind, placebo-controlled, sequential parallel study of CERC-301 
in the adjunctive treatment of subjects with severe depression and recent active suicidal ideation despite antidepressant treatment. Poster presentation at the 2015 National Network of Depression Centers Annual Conference, Nov 5-6, 2015, Ann Arbor, MI, USA.

4. Sanacora G, Johnson MR, Khan A, Atkinson SD, Riesenberg RR, Schronen JP, et al. Adjunctive lanicemine (AZD6765) in patients with major depressive disorder and history of inadequate response to antidepressants: A randomized, placebo-controlled study. Neuropsychopharmacology. 2017;42:844-53.

5. Williams NR, Heifets BD, Blasey C, Sudheimer K, Pannu J, Pankow $\mathrm{H}$, et al. Attenuation of antidepressant effects of ketamine by opioid receptor antagonism. Am J Psychiatry. 2018;175:1205-15.

6. Williams NR, Heifets BD, Bentzley BS, Blasey C, Sudheimer $\mathrm{KD}$, Hawkins J, et al. Attenuation of antidepressant and antisuicidal effects of ketamine by opioid receptor antagonism. Mol Psychiatry. 2019;24:1779-86.

7. Ryder S, Way WL, Trevor AJ. Comparative pharmacology of the optical isomers of ketamine in mice. Eur J Pharm. 1978;49:15-23.

8. Finck AD, Ngai SH. Opiate receptor mediation of ketamine analgesia. Anesthesiology. 1982;56:291-7.

9. Hustveit O, Maurset A, Oye I. Interaction of the chiral forms of ketamine with opioid, phencyclidine, sigma and muscarinic receptors. Pharm Toxicol. 1995;77:355-9.

10. Pacheco D, da F, Romero TRL, Duarte IDG. Central antinociception induced by ketamine is mediated by endogenous opioids and $\mu$ and 8-opioid receptors. Brain Res. 2014;1562:69-75.

11. Klein, ME, Chandra, J, Sheriff, S, Malinow, R. Opioid System Is Necessary but Not Sufficient for Antidepressive Actions of Ketamine in Rodents. Proceedings of the National Academy of Sciences of the United States of America. 2020;117:2656-62.
12. O’Brien B, Lijffijt M, Lee J, Kim YS, Wells A, Murphy N, et al. Distinct trajectories of antidepressant response to intravenous ketamine. J Affect Disord. 2021;286:320-9.

13. Phillips JL, Norris S, Talbot J, Birmingham M, Hatchard T, Ortiz A, et al. Single, repeated, and maintenance ketamine infusions for treatment-resistant depression: A randomized controlled trial. Am J Psychiatry. 2019;176:401-9.

14. Shiroma PR, Thuras P, Wels J, Albott CS, Erbes C, Tye S, et al. A randomized, double-blind, active placebo-controlled study of efficacy, safety, and durability of repeated vs single subanesthetic ketamine for treatment-resistant depression. Transl Psychiatry. 2020;10:206.

15. Schatzberg AF. A word to the wise about ketamine. Am J Psychiatry. 2014;171:262-4.

16. Fang Y, Wang Y, Shi J, Liu Z, Lu L. Recent trends in drug abuse in China. Acta Pharm Sin. 2006;27:140-4.

17. Sassano-Higgins S, Baron D, Juarez G, Esmaili N, Gold M. A review of ketamine abuse and diversion. Depress Anxiety. 2016;33:718-27.

18. Zhu W, Ding Z, Zhang Y, Shi J, Hashimoto K, Lu L. Risks associated with misuse of ketamine as a rapid-acting antidepressant. Neurosci Bull. 2016;32:557-64.

19. Strong CE, Kabbaj M. On the safety of repeated ketamine infusions for the treatment of depression: Effects of sex and developmental periods. Neurobiol Stress. 2018;9:166-75.

20. Zhang C, Tang WK, Liang HJ, Ungvari GS, Lin SK. Other drug use does not impact cognitive impairments in chronic ketamine users. Drug Alcohol Depend. 2018;186:1-8.

21. Zhang $\mathrm{C}$, Xu Y, Zhang B, Hao W, Tang WK. Cognitive impairment in chronic ketamine abusers. Psychiatry Res. 2020; 291:113206. 